\title{
Introduction to the special issue of the ECML PKDD 2021 journal track
}

\author{
Annalisa Appice ${ }^{1} \cdot$ Sergio Escalera ${ }^{2} \cdot$ Jose A. Gámez ${ }^{3} \cdot$ Heike Trautmann $^{4}$ \\ Published online: 27 September 2021 \\ (๑) The Author(s), under exclusive licence to Springer Science+Business Media LLC, part of Springer Nature 2021
}

The papers contained in this special issue have been accepted for the ECML PKDD 2021 journal track, which allows authors to combine a journal publication with a conference presentation of their work. The journal track was launched in 2013 and has accompanied the European Conference on Machine Learning and Knowledge Discovery in Databases (ECML PKDD) since then. It solicits high quality papers combining the timeliness and novelty of conference contributions with the maturity and sophistication of journal publications - survey papers or extensions of previously published conference papers are normally excluded. Authors can submit to the Machine Learning Journal or the Data Mining and Knowledge Discovery Journal. This year, the journal track offered four submission deadlines between September 2020 and May 2021. Accepted papers were presented (virtually) at the ECML PKDD 2021 conference in Bilbao, Spain, from September 13-17, 2021. The Machine Learning journal received a total of 107 submissions, of which 20 were accepted in time for this special issue. The special issue also contains one paper, edited by Ira Assent, Carlotta Domeniconi, Aristides Gionis, Eyke Hüllermeier, which was submitted to the journal track for ECML PKDD 2020 but not accepted in time. The contributions to this issue cover a wide spectrum of topics in machine learning, ranging from privacy and interpretability to optimization techniques in deep and variational learning. We thank all authors who submitted papers to the journal track, as well as the members of our Guest Editorial Board and other reviewers who provided timely and high-quality reviews. All papers went through a rigorous reviewing process meeting the standards of the Machine Learning Journal, and they have only been accepted after careful revision by the authors.

Annalisa Appice

annalisa.appice@uniba.it

Sergio Escalera

sergio@maia.ub.es

Jose A. Gámez

Jose.Gamez@uclm.es

Heike Trautmann

trautmann@uni-muenster.de

1 Dipartimento di Informatica, Università degli Studi di Bari “Aldo Moro", Bari, Italy

2 Universitat de Barcelona and Computer Vision Center, Barcelona, Spain

3 Universidad de Castilla-La Mancha, Albacete, Spain

4 University of Münster, Münster, Germany 
We are also grateful for the support of Hendrik Blockeel (Editor-in-Chief of the Machine Learning journal), Johannes Fürnkranz (Editor-in-Chief of the Data Mining and Knowledge Discovery journal) and Melissa Fearon (Executive Editor of Springer responsible for these journals). Finally, we thank Jose A. Lozano, the ECML PKDD 2021 general chair, and the ECML PKDD 2020 journal track chairs for their guidance throughout the year. We hope that the readers enjoy the papers in this issue.

Publisher's Note Springer Nature remains neutral with regard to jurisdictional claims in published maps and institutional affiliations. 